\title{
Risque de maladies cardiovasculaires en fonction des profils plasmatiques d'apolipoprotéines et de lipides chez une communauté des Premières nations du Canada*
}

\author{
N. D. Riediger, M. Sc. (1); S. G. Bruce, Ph. D. (1); T. K. Young, M.D., Ph. D. (2)
}

* Cet article fait l'objet d'une publication conjointe par Preventing Chronic Disease et Maladies chroniques au Canada. Preventing Chronic Disease en est le premier éditeur, Maladies chroniques au Canada le second. La publication originale est : Riediger ND, Bruce SG, Young TK. Cardiovascular risk according to plasma apolipoprotein and lipid profiles in a Canadian First Nation. Prev Chronic Dis 2011;8(1). Consultable en ligne à la page : http/www.cdc.gov/pcd/issues/2011/jan/09_0216.htm

\section{Résumé}

Introduction : Bien que le lourd fardeau du diabète chez les membres des Premières nations du Canada soit un fait connu, on en sait peu sur le risque de maladies cardiovasculaires auquel ce groupe est exposé. Notre objectif était de décrire le profil apolipoprotéique des membres d'une communauté canadienne des Premières nations et l'incidence de celui-ci sur le risque de maladies cardiovasculaires.

Méthodologie : En 2003, un échantillon représentatif d'adultes d'une Première nation du Manitoba $(\mathrm{N}=483)$ a participé à une étude de dépistage du diabète et des complications associées à cette maladie, dans le cadre de laquelle les facteurs de risque de maladies cardiovasculaires des participants ont été évalués.

Résultats : Soixante pour cent des femmes présentaient un risque cardiovasculaire accru associé à de faibles concentrations d'apolipoprotéines A1 (apo A1), comparativement à $35 \%$ des hommes. La proportion de femmes chez lesquelles on a relevé de faibles concentrations d'apo A1 diminuait avec l'âge, mais la proportion chez lesquelles de faibles taux de lipoprotéines de haute densité ont été relevés était stable dans tous les groupes d'âge. L'apo B et l'apo A1 étaient toutes deux significativement corrélées à l'obésité, une fois contrôlées les variables liées à l'âge, au sexe, à la pression artérielle diastolique, au taux d'homocystéine, au diabète et à l'insulinorésistance.

Conclusion : Les profils apolipoprotéique et lipidique de cette population des Premières nations semblent indiquer un risque de maladies cardiovasculaires élevé. Les recherches à venir devraient viser à définir la dimension des particules de lipoprotéines chez cette population.

Mots clés : cardiovasculaire, santé communautaire, diabète, épidémiologie, obésité, dépistage, minorité, Première nations, Indiens d'Amérique du Nord

\section{Introduction}

Au cours des dernières années, la prévalence des maladies cardiovasculaires chez les Autochtones du Canada a augmenté et est maintenant plus élevée que chez les non-Autochtones. Dans un échantillon
L'apolipoprotéine A1 (apo A1) est le principal constituant des lipoprotéines de haute densité (HDL), tandis que l'apo B est l'une des principales protéines composant les lipoprotéines de très basse densité, les lipoprotéines de basse densité (LDL) et les lipoprotéines de densité intermédiaire. En raison de leur association respective avec ces lipoprotéines, l'apo A1 est inversement corrélée au risque de maladies cardiovasculaires, alors que l'apo B l'est positivement ${ }^{2}$. Les données semblent d'ailleurs indiquer que l'apo A1 et l'apo B sont de meilleurs prédicteurs de risque de maladies cardiaques que le cholestérol HDL et le cholestérol $\mathrm{LDL}^{3-5}$. Les mesures des apolipoprotéines pourraient également offrir des avantages par rapport à celle des lipoprotéines, puisqu'elles sont mesurées directement, contrairement au cholestérol LDL, par exemple, qui doit être mesuré à partir d'autres lipoprotéines à l'aide d'un échantillon sanguin prélevé à jeun.

Malgré les taux élevés de diabète et de maladies cardiovasculaires chez les Autochtones canadiens, peu de chercheurs se sont aventurés au-delà de l'examen des facteurs de risque classiques. De plus, les recherches étaient généralement fondées sur l'analyse des dossiers, et les quelques études en population qui ont été réalisées étaient limitées à une seule communauté des Premières nations ${ }^{6-8}$. Notre objectif était de décrire le profil apolipoprotéique et ses liens avec les facteurs de risque de maladies cardiovasculaires chez une communauté des Premières nations.

Rattachement

1. Université du Manitoba, Winnipeg (Manitoba) Canada

2. Université de Toronto, Toronto (Ontario) Canada

Correspondance : Natalie D. Riediger, M. Sc., Université du Manitoba, bureau S113, Medical Services Bldg, 750 Bannatyne Ave, Winnipeg (Manitoba) Canada R3E 0W3; tél. : 204-975-7745; courriel : umriedin@cc.umanitoba.ca 


\section{Méthodologie}

La présente étude s'est appuyée sur des données recueillies dans le cadre d'une vaste étude de dépistage portant sur les complications associées au diabète ${ }^{9}$. L'échantillon, composé de 483 hommes et femmes d'une communauté des Premières nations du Manitoba, était représentatif en ce qui a trait à l'âge et au sexe. Les participants admissibles ( $\mathrm{n}=1$ 356) étaient des hommes et des femmes (non enceintes) de 18 ans ou plus, possédant le statut d'Indien inscrit et vivant dans la communauté. Celle-ci se situe à environ $200 \mathrm{~km}$ au nord-ouest de Winnipeg (Manitoba). L'étude a été réalisée entre janvier et décembre 2003, et les données pour chacun des participants ont été recueillies au cours de la même journée. Les détails au sujet de l'étude sont décrits ailleurs ${ }^{9}$. Cette étude a été approuvée par le conseil d'éthique de la recherche en santé de l’Université du Manitoba.

Une infirmière autorisée a prélevé des échantillons de sang à jeun, à partir desquels les concentrations plasmatiques en glucose, en insuline, en triglycérides, en cholestérol HDL, en cholestérol LDL, en cholestérol total, en apo B totale et en apo A1 ont été mesurées. L'infirmière a également mesuré la pression artérielle, le taux d'albumine et de créatinine dans les urines, et les caractéristiques anthropométriques de chaque participant ${ }^{9}$. Nous avons utilisé le modèle d'évaluation homéostatique (HOMA), qui permet, à partir des mesures de glucose et d'insuline, d'évaluer l'insulinorésistance en appliquant la formule suivante : (insuline $[\mathrm{pmol}] \times 0,139) \times($ glucose $[\mathrm{mmol} / \mathrm{L}] / 22,5)$. Les facteurs de risque évalués dans cette étude figurent au tableau 1.

Toutes les données ont été analysées à l'aide du logiciel SPSS version 16.0 pour Windows (IBM, Chicago, Illinois). Nous avons comparé les concentrations plasmatiques en lipides à l'aide du test $t$ et du test $\mathrm{U}$ de Mann-Whitney (non paramétrique). Pour les variables qui ne suivent pas une distribution normale ou pour les cas de variance inégale, nous avons comparé les différences entre les concentrations en apolipoprotéines pour
TABLEAU 1

Facteurs de risque évalués dans une étude sur les facteurs de risque de maladies cardiovasculaires réalisée auprès d'une communauté des Premières nations du Canada, 2003

\begin{tabular}{|c|c|c|}
\hline \multirow{2}{*}{ Facteur de risque } & \multicolumn{2}{|c|}{ Définition } \\
\hline & Hommes & Femmes \\
\hline Obésité & IMC $\geq 30,0 \mathrm{~kg} / \mathrm{m}^{2}$ & \\
\hline $\begin{array}{l}\text { TT associé } \\
\text { à un risque élevé }\end{array}$ & $\mathrm{TT}>102 \mathrm{~cm}$ & $\mathrm{TT}>88 \mathrm{~cm}$ \\
\hline Diabète & \multicolumn{2}{|c|}{$\begin{array}{l}\text { Diagnostic autodéclaré, prise d'un hypoglycémiant oral ou gilycémie } \\
\text { à jeun } \geq 7,0 \mathrm{mmol} / \mathrm{L}\end{array}$} \\
\hline Hypertension & \multicolumn{2}{|c|}{ Diagnostic autodéclaré, PAS $>140 \mathrm{~mm} \mathrm{Hg} \mathrm{ou} \mathrm{PAD}>90 \mathrm{~mm} \mathrm{Hg}$} \\
\hline Dyslipidémie & $\begin{array}{l}\text { TG plasmatiques à jeun } \geq 1,7 \mathrm{mmol} / \mathrm{L} \\
\text { et cholestérol } \mathrm{HDL} \text { plasmatique à } \\
\text { jeun } \leq 1,03 \mathrm{mmol} / \mathrm{L}\end{array}$ & $\begin{array}{l}\text { TG plasmatiques à jeun } \geq 1,7 \mathrm{mmol} / \mathrm{L} \\
\text { et cholestérol } \mathrm{HDL} \text { plasmatique à jeun } \\
\leq 1,3 \mathrm{mmol} / \mathrm{L}\end{array}$ \\
\hline Microalbuminurie $^{\mathrm{a}}$ & $\mathrm{RAC}>2,0 \mathrm{mg} / \mathrm{mmol}$ & $\mathrm{RAC}>2,8 \mathrm{mg} / \mathrm{mmol}$ \\
\hline Syndrome métabolique & \multicolumn{2}{|l|}{ Critères du Adult Treatment Panel III ${ }^{10}$} \\
\hline Risque cardiométabolique & \multicolumn{2}{|c|}{ TT à risque et TG plasmatiques $\geq 1,7 \mathrm{mmol} / \mathrm{L}$} \\
\hline Apo A1 faible & ApoA1 $<1,07 \mathrm{~g} / \mathrm{L}$ & ApoA1 $<1,22 \mathrm{~g} / \mathrm{L}$ \\
\hline Apo B élevée & ApoB $>1,2 \mathrm{~g} / \mathrm{L}$ & \\
\hline $\begin{array}{l}\text { Rapport apo B-apo } \\
\text { A1 élevé }{ }^{5}\end{array}$ & $>0,8$ & $>0,7$ \\
\hline
\end{tabular}

Abréviations : IMC, indice de masse corporelle; TT, tour de taille; PAS, pression artérielle systolique; PAD, pression artérielle diastolique; TG, triglycérides; HDL, lipoprotéines de haute densité; RAC, rapport albumine-créatinine; apo, apolipoprotéine.

a Déterminé à l’aide du Bayer DCA 2000 point-of-care analyzer (Elkhart, Indiana).

TABLEAU 2

Concentrations plasmatiques en lipides chez 483 adultes d'une communauté des Premières nations du Canada, 2003

\begin{tabular}{|c|c|c|c|c|c|c|c|}
\hline \multirow{3}{*}{ Triglycérides, mmol/L } & \multicolumn{2}{|c|}{$\begin{array}{c}\text { Hommes } \\
(n=230), \\
\text { moyenne (ET) }\end{array}$} & \multicolumn{2}{|c|}{$\begin{array}{c}\text { Femmes } \\
(\mathrm{n}=253), \\
\text { moyenne (ET) }\end{array}$} & \multirow{3}{*}{$\begin{array}{c}\text { Valeur } \boldsymbol{p}^{\mathbf{b}} \\
0,86\end{array}$} & \multicolumn{2}{|c|}{$\begin{array}{l}\text { Hommes } \\
\text { et femmes, } \\
\text { moyenne (ET) }\end{array}$} \\
\hline & 2,3 & $(2,5)$ & 2,1 & $(2,0)$ & & 2,2 & $(2,3)$ \\
\hline & 1,7 & $(1,1-2,6)^{\mathrm{c}}$ & 1,7 & $(1,2-2,5)^{c}$ & & 1,7 & $2-2,6)^{c}$ \\
\hline Cholestérol LDL, mmol/L & 2,9 & $(0,9)$ & 2,6 & $(0,9)$ & $<0,001$ & 2,7 & $(0,9)$ \\
\hline Cholestérol HDL, mmol/L & 1,2 & $(0,3)$ & 1,2 & $(0,3)$ & $<0,04^{d}$ & 1,2 & $(0,3)$ \\
\hline Cholestérol total, mmol/L & 5,0 & $(1,2)$ & 4,8 & $(1,1)$ & 0,07 & 4,9 & $(1,2)$ \\
\hline
\end{tabular}

Abréviations : ET, écart-type; LDL, lipoprotéines de basse densité; HDL, lipoprotéines de haute densité.

${ }^{a}$ Les taux moyens de cholestérol LDL et de cholestérol total sont indiqués, même si ces valeurs ne font pas partie de la définition de la dyslipidémie, puisque leur niveau recommandé varie en fonction d'autres facteurs de risque (http://www.cfpc.ca/French/cfpc/programs/patient\%20education/cholesterol/default.asp).

${ }^{\mathrm{b}}$ Test $\mathrm{t}$ indépendant utilisé pour déterminer les différences entre les sexes, à moins d'indication contraire.

' Les données présentées sont une médiane (intervalle interquartile) en raison de la distribution asymétrique; analyse statistique réalisée à l'aide du test de Mann-Whitney.

${ }^{d}$ Test de Mann-Whitney (variances inégales). 
TABLEAU 3

Concentrations plasmatiques d'apolipoprotéines selon le sexe et les facteurs de risque de maladies cardiovasculaires chez 483 adultes d'une communauté des Premières nations du Canada, 2003a

\begin{tabular}{|c|c|c|c|c|c|c|c|c|c|c|}
\hline Caractéristique $^{b}$ & n (\%) & \multicolumn{2}{|c|}{ ApoB (g/L) } & Valeur $\boldsymbol{p}$ & \multicolumn{2}{|c|}{ ApoA1 (g/L) } & Valeur $p$ & \multicolumn{2}{|c|}{$\begin{array}{c}\text { ApoB:ApoA1 } \\
\text { Ratio }\end{array}$} & Valeur $p$ \\
\hline \multicolumn{11}{|l|}{ Sexe } \\
\hline Hommes & $230(48)$ & 0,94 & $(0,28)$ & \multirow{2}{*}{0,046} & \multirow{2}{*}{\multicolumn{2}{|c|}{$\begin{array}{l}1,14(1,03-1,23)^{c} \\
1,17(1,05-1,31)^{c}\end{array}$}} & \multirow{2}{*}{$0,004^{d}$} & 0,84 & $3-1,02)^{c}$ & \multirow{2}{*}{$0,001^{d}$} \\
\hline Femmes & $253(52)$ & 0,89 & $(0,26)$ & & & & & 0,75 & $9-0,91)^{c}$ & \\
\hline \multicolumn{11}{|l|}{ Obésité } \\
\hline Oui & $265(56)$ & 0,97 & $(0,96)$ & \multirow{2}{*}{$<0,001$} & 1,13 & $(0,17)$ & \multirow{2}{*}{$<0,001$} & 0,87 & $(0,24)$ & \multirow{2}{*}{$<0,001$} \\
\hline Non & $204(44)$ & 0,83 & $(0,79)$ & & 1,20 & $(0,19)$ & & 0,71 & $(0,24)$ & \\
\hline \multicolumn{11}{|c|}{ Tour de taille associé à un risque } \\
\hline Oui & $313(68)$ & 0,96 & $(0,26)$ & \multirow{2}{*}{$<0,001$} & 1,15 & $(0,18)$ & \multirow{2}{*}{0,006} & 0,85 & $(0,25)$ & \multirow{2}{*}{$<0,001$} \\
\hline Non & $151(32)$ & 0,82 & $(0,26)$ & & 1,20 & $(0,18)$ & & 0,70 & $(0,23)$ & \\
\hline \multicolumn{11}{|l|}{ Diabète } \\
\hline Oui & 140 (29) & 1,05 & $(0,29)$ & \multirow{2}{*}{$<0,001$} & 1,16 & $(0,19)$ & \multirow{2}{*}{0,92} & 0,91 & $(0,26)$ & \multirow{2}{*}{$<0,001$} \\
\hline Non & 343 (71) & 0,86 & $(0,25)$ & & 1,17 & $(0,18)$ & & 0,75 & $(0,23)$ & \\
\hline \multicolumn{11}{|l|}{ Hypertension } \\
\hline Oui & $201(43)$ & 0,99 & $(0,28)$ & \multirow{2}{*}{$<0,001$} & 1,18 & $(0,19)$ & \multirow{2}{*}{0,10} & 0,85 & $(0,27)$ & \multirow{2}{*}{$<0,001$} \\
\hline Non & 271 (57) & 0,86 & $(0,25)$ & & 1,15 & $(0,18)$ & & 0,76 & $(0,23)$ & \\
\hline \multicolumn{11}{|l|}{ Microalbuminurie } \\
\hline Oui & $94(20)$ & 1,01 & $1-1,26)^{c}$ & \multirow{2}{*}{$<0,001^{\mathrm{d}}$} & 1,15 & $(0,17)$ & \multirow{2}{*}{0,49} & 0,90 & $(0,26)$ & \multirow{2}{*}{$<0,001$} \\
\hline Non & 372 (80) & 0,86 & $8-1,05)^{c}$ & & 1,17 & $(0,18)$ & & 0,77 & $(0,24)$ & \\
\hline \multicolumn{11}{|c|}{ Risque cardiométabolique } \\
\hline Oui & $212(45)$ & 1,05 & $(0,25)$ & \multirow{2}{*}{$<0,001$} & 1,16 & $(0,19)$ & 054 & 0,93 & $(0,24)$ & \\
\hline Non & $255(55)$ & 0,80 & $(0,23)$ & & 1,17 & $(0,18)$ & 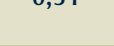 & 0,70 & $(0,21)$ & 0,001 \\
\hline Dyslipidémie & & & & & & & & & & \\
\hline Oui & $155(32)$ & 1,03 & $0-1,19)^{c}$ & 0 001d & 1,07 & $8-1,18)^{c}$ & 0 0 & 0,98 & $(0,23)$ & - \\
\hline Non & $328(68)$ & 0,81 & $5-1,01)^{c}$ & 0,001 & 1,19 & $9-1,31)^{c}$ & 0,001 & 0,72 & $(0,22)$ & 0,001 \\
\hline Syndrome métabo & & & & & & & & & & \\
\hline Oui & $252(53)$ & 1,02 & $(0,26)$ & 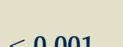 & 1,13 & $(0,17)$ & 0001 & 0,91 & $5-1,05)^{c}$ & \\
\hline Non & $223(47)$ & 0,80 & $(0,23)$ & $-0,001$ & 1,20 & $(0,18)$ & $-0,001$ & 0,64 & $3-0,80)^{c}$ & \\
\hline
\end{tabular}

Abréviations : apo, apolipoprotéine.

${ }^{a}$ Les valeurs présentées pour l'apo A1 et l'apo B et le rapport apo B-apo A1 sont une moyenne (écart-type), et les différences ont été évaluées à l'aide du test t sur des échantillons indépendants, à moins d'indication contraire.

${ }^{\mathrm{b}}$ Les définitions des caractéristiques figurent au tableau 1. Les données pour certaines caractéristiques n'étaient pas disponibles pour certains participants.

' Les données présentées sont une médiane (intervalle interquartile) en raison de la distribution asymétrique.

d Test U de Mann-Whitney (non paramétrique).

chaque facteur de risque de maladies cardiovasculaires à l'aide du test $t$ et du test U de Mann-Whitney. Nous avons utilisé le test $\chi^{2}$ pour déceler les différences entre les risques de maladies cardiovasculaires pour chaque catégorie d'apolipoprotéine. Les tests étaient bilatéraux, et les valeurs $p<0,05$ ont été jugées significatives. Afin de déterminer les tendances linéaires pour les valeurs moyennes d'apolipoprotéines de chaque groupe d'âge, nous avons utilisé l'analyse de variance à un facteur avec contraste linéaire. Nous avons estimé les rapports de cote pour l'obésité à l'aide du modèle de régression logistique multivariée descendante. Nous avons inclus, dans le modèle, des variables qui avaient été significativement corrélées avec l'obésité au moyen d'analyses bivariées. Ces variables étaient l'âge, le sexe, le tabagisme (présent ou passé), la pression artérielle systolique et diastolique, le diabète, le taux de triglycérides, les concentrations d'apo A1 et d'apo B, l'insulinorésistance, le taux d'homocystéine et la microalbuminurie.

\section{Résultats}

Le risque de maladies cardiovasculaires chez la population à l'étude était élevé si l'on se fie aux facteurs de risque classiques tels que les taux de cholestérol HDL et de triglycérides (tableau 2). Les taux d'obésité, de diabète, d'hypertension et de microalbuminurie étaient également élevés (tableau 3).

Il y avait significativement plus de femmes que d'hommes $(60 \%$ c. $35 \%$; 
TABLEAU 4

Concentrations plasmatiques d'apolipoprotéines selon le sexe et l'âge chez 481a adultes d'une communauté des Premières nations du Canada, 2003

\begin{tabular}{|c|c|c|c|c|c|c|}
\hline Sexe et âge (ans) & moyenne (ET) apo B & Valeur $\boldsymbol{p}^{\mathrm{b}}$ & $\begin{array}{c}\text { moyenne (ET) } \\
\text { apo A1 }\end{array}$ & Valeur $\boldsymbol{p}^{\mathrm{b}}$ & $\begin{array}{c}\text { moyenne }(\mathrm{ET}) \\
\text { rapport apoB } \\
\text { apo } \mathrm{A1}\end{array}$ & Valeur $p^{b}$ \\
\hline \multicolumn{7}{|l|}{ Hommes $(n=229)$} \\
\hline $18-29(n=72)$ & $0,79(0,26)$ & \multirow{4}{*}{$<0,001$} & $1,12(0,14)$ & \multirow{4}{*}{0,12} & $0,71(0,25)$ & \multirow{4}{*}{$<0,001$} \\
\hline $30-39(n=65)$ & $0,95(0,25)$ & & $1,14(0,15)$ & & $0,85(0,25)$ & \\
\hline $40-49(n=49)$ & $1,09(0,26)$ & & $1,13(0,16)$ & & $0,97(0,24)$ & \\
\hline$\geq 50(n=43)$ & $1,01(0,25)$ & & $1,17(0,17)$ & & $0,88(0,24)$ & \\
\hline \multicolumn{7}{|l|}{ Femmes $(n=252)$} \\
\hline $18-29(n=70)$ & $0,78(0,24)$ & \multirow{4}{*}{$<0,001$} & $1,15(0,20)$ & \multirow{4}{*}{0,03} & $0,69(0,22)$ & \multirow{4}{*}{0,001} \\
\hline $30-39(n=78)$ & $0,89(0,22)$ & & $1,20(0,17)$ & & $0,75(0,21)$ & \\
\hline $40-49(n=59)$ & $0,96(0,27)$ & & $1,21(0,21)$ & & $0,81(0,25)$ & \\
\hline$\geq 50(n=45)$ & $0,99(0,29)$ & & $1,22(0,22)$ & & $0,83(0,28)$ & \\
\hline
\end{tabular}

Abréviations : é.t., écart-type; apo, apolipoprotéine.

${ }^{a}$ L'échantillon sanguin de deux participants était insuffisant pour évaluer la concentration en apo A1 et en apo B; les autres lipides plasmatique (cholestérol total, cholestérol LDL, cholestérol HDL et triglycérides) ont été mesurés en priorité.

${ }^{\mathrm{b}}$ Analyse de la variance avec contraste linéaire.

TABLEAU 5

Adultes d'une communauté des Premières nations du Canada à risque de maladies cardiovasculaires en fonction des concentrations plasmatiques d'apolipoprotéines, $2003(\mathrm{~N}=481)^{\mathrm{a}}$

\begin{tabular}{|c|c|c|c|c|c|c|}
\hline Sexe et âge (ans) & n (\%) аров & Valeur $\mathbf{p}^{\mathbf{b}}$ & n (\%) аро A1 & Valeur $\mathbf{p}^{\mathbf{b}}$ & $\begin{array}{l}\text { n (\%) rapport } \\
\text { apo B-apo A1 }\end{array}$ & Valeur $\mathbf{p}^{\mathrm{b}}$ \\
\hline \multicolumn{7}{|l|}{ Hommes $(n=229)$} \\
\hline $18-29(n=72)$ & $5(7)$ & \multirow{4}{*}{0,003} & $25(35)$ & \multirow{4}{*}{0,87} & $20(29)$ & \multirow{4}{*}{$<0,001$} \\
\hline $30-39(n=65)$ & $10(15)$ & & $24(37)$ & & $37(57)$ & \\
\hline $40-49(n=49)$ & $15(31)$ & & $18(37)$ & & $40(82)$ & \\
\hline$\geq 50(n=43)$ & $10(23)$ & & $14(33)$ & & $25(58)$ & \\
\hline \multicolumn{7}{|l|}{ Femmes $(n=252)$} \\
\hline $18-29(n=70)$ & $5(7)$ & \multirow{4}{*}{0,006} & $52(74)$ & \multirow{4}{*}{0,006} & $30(43)$ & \multirow{4}{*}{0,01} \\
\hline $30-39(n=78)$ & $6(8)$ & & $43(55)$ & & $45(58)$ & \\
\hline $40-49(n=59)$ & $10(17)$ & & $37(63)$ & & $38(65)$ & \\
\hline$\geq 50(n=45)$ & $10(22)$ & & 20 (44) & & $29(64)$ & \\
\hline
\end{tabular}

Abréviations : apo, apolipoprotéine.

${ }^{a}$ Les seuils de coupure pour le risque de maladies cardiovasculaires accru sont présentés dans le tableau 1. L'échantillon sanguin de deux participants était insuffisant pour évaluer la concentration en apo A1 et en apo B; les autres lipides du plasma (cholestérol total, cholestérol LDL, cholestérol HDL et triglycérides) ont été mesurés en priorité.

${ }^{\mathrm{b}}$ Test $\chi^{2}$ avec association linéaire.

$p<0,001)$ qui présentaient des concentrations d'apo A1 associées à un risque de maladies cardiovasculaires. Près de $18 \%$ des hommes et $12 \%$ des femmes avaient des concentrations d'apo $\mathrm{B}$ associées à un risque de maladies cardiovasculaires, mais la différence n'était pas significative. La proportion des participants dont le rapport apo B-apo A1 révélait un risque accru était de $54 \%$ chez les hommes et de $57 \%$ chez les femmes, mais cette différence n'a pas atteint le seuil de signification.
Les concentrations moyennes d'apo B et le rapport apo B-apo A1 étaient significativement plus élevés chez les hommes et chez les participants présentant un quelconque facteur de risque cardiovasculaire (tableau 3). Les concentrations moyennes d'apo A1 étaient moins élevées chez les patients présentant la plupart des facteurs de risque de maladies cardiovasculaires, mais l'écart n'a pas atteint le seuil de signification chez les patients souffrant de diabète, d'hypertension ou de microalbuminurie, ou ayant des risques cardiométaboliques.
Le risque de maladies cardiovasculaires tendait à augmenter avec l'âge (tableaux 4 et 5). Chez les hommes, nous avons observé une tendance linéaire significative dans l'association entre l'âge et le taux d'apo B et le rapport apo B-apo A1. Nous avons également noté une tendance linéaire positive significative dans l'association entre l'âge des femmes et le taux d'apo B et le rapport apo B-apo A1. Inversement, le risque de maladies cardiovasculaires en fonction du taux d'apo A1 diminuait avec l'âge chez les femmes, et 
les concentrations moyennes d'apo A1 augmentaient avec l'âge. Chez les femmes, les taux de cholestérol HDL n'ont ni augmenté ni diminué de façon significative avec l'âge (données non présentées).

Dans les cas d'obésité, le modèle logistique final a inclus l'âge, le sexe, la pression artérielle diastolique, la présence ou non de diabète, le taux d'homocystéine, l'insulinorésistance et les concentrations d'apo A1 et d'apo B. Un sujet chez qui la concentration d'apo A1 était de 1,14 g/l était 1,2 fois plus susceptible d'être obèse qu'un sujet chez qui la concentration d'apo A1 était de 1,20 g/l. De plus, la probabilité d'obésité était 1,35 fois plus élevée pour un sujet chez qui la concentration en apo B était de $1,00 \mathrm{~g} / \mathrm{l}$ que pour une personne chez qui cette concentration était de 0,80 g/l.

\section{Analyse}

Le risque de maladies cardiovasculaires est élevé chez les membres des Premières nations du Canada, si l'on se fie aux profils plasmatiques de lipides et d'apolipoprotéines. Les concentrations plasmatiques en apolipoprotéines anormales qui ont été relevées concordent avec la prévalence de l'obésité et du diabète dans cette communauté. En règle générale, les taux de cholestérol HDL et d'apo A1 des participants étaient bas, tandis que leur taux de triglycérides était élevé, ce qui est normalement le cas chez les personnes insulinorésistantes.

Le profil lipidique moyen de la communauté à l'étude différait considérablement de celui de la population des É.-U. en général ${ }^{11}$, vraisemblablement en raison de la prévalence élevée du diabète dans ce groupe. Par exemple, les taux plasmatiques moyens de cholestérol LDL des participants de la présente étude étaient moins élevés que ceux des participants de la NHANES*, et ce, pour les deux sexes. Cependant, les taux plasmatiques de cholestérol HDL étaient moins élevés et les taux plasmatiques de triglycérides étaient beaucoup plus élevés chez les sujets de notre étude que dans la population américaine en général. Comparativement aux Aborigènes d'Australie et aux habitants du détroit de Torres ${ }^{12}$, les taux de triglycérides

\footnotetext{
* National Health and Nutrition Examination Survey
}

chez les participants de la présente étude étaient légèrement inférieurs et les taux de cholestérol HDL, légèrement supérieurs. Par contre, ces taux sont tout de même pires que ceux des Autochtones crisojibwés, des Inuits et des non-Autochtones du Canada, d'après une étude réalisée au début des années $1990^{13}$. De plus, les taux de triglycérides et de cholestérol HDL étaient plus élevés, et les taux de cholestérol LDL moins élevés, chez la communauté à l'étude que chez une autre communauté des Premières nations du Canada ${ }^{1}$; cependant, les participants de cette autre communauté autochtone étaient âgés de 35 à 75 ans, tandis que ceux de notre étude étaient âgées de 18 ans ou plus (avec une moyenne d'âge de 38 ans). Ni les valeurs lipidiques ni les autres valeurs présentées dans ce paragraphe n'ont été normalisées en fonction de l'âge ou du sexe.

Paradoxalement, la proportion des femmes qui présentaient de faibles concentrations d'apo A1 (associées à un risque de maladies cardiovasculaires élevé) diminuait significativement avec l'âge, et l'on observait une tendance linéaire positive significative dans l'association entre les concentrations moyennes d'apo A1 et l'âge. La variation des concentrations d'apo A1 en fonction de l'âge chez les femmes semble indiquer une prévalence plus élevée de la dyslipidémie chez les jeunes femmes. Cependant, la proportion de femmes affichant un taux de cholestérol HDL associé à un risque élevé est demeurée stable dans tous les groupes d'âge. Pour un même taux de cholestérol HDL, les femmes âgées ( $\geq 50$ ans) présentaient des concentrations d'apo A1 moins élevées que les femmes plus jeunes ( $<50$ ans). Ce phénomène pourrait s'expliquer par un changement dans la taille des particules de HDL qui se produit chez les groupes d'âge plus avancé, c'est-à-dire que la proportion des petites particules denses de HDL augmente par rapport à celle des particules de HDL de grande taille. Le fait que, dans le cadre de notre étude, la prévalence du diabète augmentait avec l'âge vient appuyer cette explication.

Malgré l'existence vraisemblable d'une prédisposition génétique au diabète et à ses troubles concomitants chez la communauté des Premières nations à l'étude, nous supposons que la majeure partie des cas de dyslipidémie sont attribuables à une mauvaise alimentation et à l'inactivité physique. Par le passé, d'autres communautés autochtones, tels les Inuits du Groenland, présentaient en fait des profils lipidiques plus avantageux que les non-Autochtones, probablement en raison de leur mode de vie traditionnel ${ }^{14}$. En effet, comparativement à un groupe témoin de Danois, les Inuits affichaient des concentrations d'apo A1 significativement plus élevées et des taux d'apo B, de cholestérol LDL, de cholestérol total et de triglycérides significativement plus bas. Même si les concentrations en apo A1 étaient significativement supérieures chez les Inuits, les taux de cholestérol HDL quant à eux ne l'étaient pas, ce qui porte à croire qu'il pourrait exister des différences (liées à la taille) entre les types de particules de HDL présentes chez ces deux groupes. Il est possible que les Inuits aient un nombre disproportionné de petites particules denses de HDL à caractère athérogène par rapport au nombre de particules de HDL de grande taille, qui sont bénéfiques pour la santé. Nous croyons que cette hypothèse pourrait également s'appliquer à la communauté qui a fait l'objet de notre étude, particulièrement aux femmes. De plus, chez une communauté de la Première nation oji-crie du Canada, même si les concentrations en apo A1 étaient significativement plus basses chez les hommes ayant un tour de taille hypertriglycéridémique, ces concentrations étaient en fait plus élevées, mais de manière non significative, chez les femmes présentant la même caractéristique ${ }^{15}$. Cette différence quant à l'apo A1 entre les sexes pourrait expliquer, en partie, le fait que les femmes atteintes de diabète sont davantage à risque de coronaropathie que les hommes souffrant de la même maladie ${ }^{16}$.

Une prépondérance de petites particules denses de LDL et de petites particules de HDL est associée à l'obésité ${ }^{17}$, à un risque accru de coronaropathie $^{18}$ et à l'insulinorésistance, qu'il y ait ou non présence de diabète ${ }^{19}$. La réduction spécifique des particules de HDL de grande taille (ainsi que l'augmentation possible des particules de HDL de petite taille), 
ne résultant pas nécessairement en une réduction significative des concentrations en apo A1, pourrait expliquer les faibles taux de cholestérol HDL chez les diabétiques $^{19}$. Dans le cadre de notre étude, la forte prévalence du diabète nous a amenés à penser que les faibles taux de cholestérol HDL relevés pouvaient être attribuables à la chute du nombre de particules de HDL de grande taille, particulièrement chez les femmes âgées. De plus, les taux de cholestérol HDL dans les cas d'obésité pourraient être moins élevés chez les femmes que chez les hommes ${ }^{20}$, et les concentrations en apo A1 pourraient donc également différer entre les deux sexes en réaction à l'obésité.

Les résultats apparemment contradictoires obtenus pour les taux d'apo A1 et de cholestérol HDL pourraient avoir une incidence sur le rôle que jouent l'apo A1 et le rapport apo B-apo A1 dans la prédiction du risque de maladies cardiovasculaires chez cette population. Les recherches à venir devraient viser à déterminer les effets sur la santé cardiovasculaire de la concentration d'apo A1 et du rapport apo B-apo A1 chez les Autochtones souffrant de diabète. Le rapport apo B-apo A1 s'est révélé un facteur prédictif du syndrome métabolique chez les participants non obèses, mais pas chez les participants obèses ${ }^{21}$. Cela pourrait s'expliquer par le fait que les personnes obèses sont davantage sujettes au diabète. Les valeurs moyennes pour l'apo A1 relevées chez les participants diabétiques de la communauté à l'étude étaient pratiquement identiques à celles des patients non diabétiques, mais les taux moyens de cholestérol HDL étaient significativement inférieurs chez les participants atteints de diabète. Cette constatation vient appuyer l'hypothèse selon laquelle le profil de HDL des membres de cette communauté est essentiellement composé de particules de petite taille, ce qui maintiendrait les taux plasmatiques de HDL peu élevés mais ferait augmenter les concentrations d'apo A1.

Notre étude comporte plusieurs limites. Même si nous ne sommes pas en mesure de déterminer si l'échantillon était entièrement représentatif de la population, nous pouvons affirmer qu'il l'était sur le plan de l'âge et du sexe, et qu'il n'était pas constitué de sujets affichant le pire état de santé. Seuls 105 des 275 membres de la communauté ayant préalablement reçu un diagnostic de diabète ont participé à l'étude. De plus, seulement 3 des 10 membres de la communauté ayant subi une amputation et aucun des 15 membres souffrant d'insuffisance rénale terminale ont collaboré avec nous. Nous n'avons pas évalué la taille des particules de lipoprotéines ni la répartition de la taille des particules selon le type de lipoprotéine. En raison de la nature transversale des données, nous n'avons pu produire aucun résultat en terme d'équation.

En conclusion, les profils plasmatiques des lipides tout comme ceux des apolipoprotéines chez les membres de la communauté à l'étude révèlent un risque de maladies cardiovasculaires élevé. La majeure partie du risque est attribuable au nombre élevé de personnes souffrant de diabète et d'obésité et à la modification du profil lipoprotéique associée à ces facteurs. Il nous faut davantage de données sur la taille des particules de lipoprotéines et sur la répartition des particules de HDL et de LDL de petite, moyenne et grande taille afin de confirmer notre hypothèse. Les données préliminaires recueillies peuvent tout de même être utilisées pour orienter les interventions visant à réduire la prévalence des maladies chroniques chez les Autochtones canadiens.

\section{Remerciements}

Cette recherche a été appuyée financièrement par les Instituts de recherche en santé du Canada (IRSC) et le Conseil manitobain de la recherche en matière de santé. Natalie Riediger est titulaire d'une bourse d'études doctorales des Instituts de recherche en santé du Canada.

\section{Références}

1. Anand SS, Yusef S, Jacobs R, Davis AD, Yi Q, Gerstein $\mathrm{H}$, et al. Risk factors, atherosclerosis, and cardiovascular disease among aboriginal people in Canada: the Study of Health Assessment and Risk Evaluation in Aboriginal Peoples (SHARE-AP). Lancet 2001;358(9288):1147-53.
2. Walldius G, Jungner I. The apoB/apoA-I ratio: a strong, new risk factor for cardiovascular disease and a target for lipidlowering therapy - a review of the evidence. J Intern Med 2006;259:493-519.

3. Walldius G, Jungner I, Holme I, Aastveit AH, Kolar W, Steiner E. High apolipoprotein B, low apolipoprotein A-I, and improvement in the prediction of fatal myocardial infarction (AMORIS study): a prospective study. Lancet 2001;358:2026-33.

4. Garfagnini A, Devoto G, Rosselli P, Boggiano P, Venturini M. Relationship between HDL-cholesterol and apolipoprotein A1 and the severity of coronary artery disease. Eur Heart J 1995;16(4):465-70.

5. Walldius G, Jungner I, Aastveit AH, Holme I, Furberg CD, Sniderman AD. The apoB/ apoA-I ratio is better than the cholesterol ratios to estimate the balance between plasma proatherogenic and antiatherogenic lipoproteins and to predict coronary risk. Clin Chem Lab Med 2004;42(12):1355-63.

6. Retnakaran R, Hanley AJ, Connelly PW, Harris SB, Zinman B. Cigarette smoking and cardiovascular risk factors among aboriginal Canadian youths. CMAJ 2005;173(8):885-9.

7. Harris SB, Zinman B, Hanley A, Gittelsohn J, Hegele R, Connelly PW, et al. The impact of diabetes on cardiovascular risk factors and outcomes in a native Canadian population. Diabetes Res Clin Pract 2002;55(2):165-73.

8. Ley SH, Harris SB, Mamakeesick M, Noon T, Fiddler E, Gittelsohn J, et al. Metabolic syndrome and its components as predictors of incident type 2 diabetes mellitus in an aboriginal community. CMAJ 2009;180(6):617-24.

9. Bruce SG, Young TK. Prevalence and risk factors for neuropathy in a Canadian First Nation community. Diabetes Care 2008;31(9):1837-41. 
10. Expert Panel on Detection, Evaluation, and Treatment of High Blood Cholesterol in Adults. Executive summary of the Third Report of the National Cholesterol Education Program (NCEP) Expert Panel on Detection, Evaluation, and Treatment of High Blood Cholesterol in Adults (Adult Treatment Panel III). JAMA 2001;285(19):2486-97.

11. Ghandehari H, Kamal-Bahl S, Wong ND. Prevalence and extent of dyslipidemia and recommended lipid levels in US adults with and without cardiovascular comorbidities: the National Health and Nutrition Examination Survey 2003-2004. Am Heart J 2008;156:112-9.

12. Wang Z, Rowley K, Wang Z, Piers L, O’Dea K. Anthropometric indices and their relationship with diabetes, hypertension and dyslipidemia in Australian Aboriginal people and Torres Strait Islanders. Eur J Cardiovasc Prev Rehabil 2007;14(2):172-8.

13. Kue Young T, Chateau D, Zhang M. Factor analysis of ethnic variation in the multiple metabolic (insulin resistance) syndrome in 3 Canadian populations. Am J Hum Biol 2002;14(5):649-58.

14. Gerdes LU, Schmidt EB, Klausen IC, Kristensen SD, Ernst E, Faergeman O, et al. Plasma concentration levels of apolipoprotein A-I, apolipoprotein B and lipoprotein (a) in Greenland Inuit (Eskimos). J Intern Med 1992;231:623-5.

15. Pollex RL, Hanley AJ, Zinman B, Harris SB, Hegele RA. Clinical and genetic associations with hypertriglyceridemic waist in a Canadian aboriginal population. Int J Obes (Lond) 2006;30(3):484-91.

16. Wang Z, Hoy WE. Association between diabetes and coronary heart disease in aboriginal people: are women disadvantaged? Med J Aust 2004;180(10):508-11.

17. Magkos F, Mohammed BS, Mittendorfer B. Effect of obesity on the plasma lipoprotein profile in normoglycemic and normolipidemic men and women. Int J Obes (Lond) 2008;32(11):1655-64.
18. El Harchaoui K, Arsenault BJ, Franssen R, Després JP, Hovingh GK, Stroes ES, et al. High-density lipoprotein particle size and concentration and coronary risk. Ann Intern Med 2009;150(2):84-93.

19. Garvey WT, Kwon S, Zheng D, Shaughnessy S, Wallace P, Hutto A, et al. Effects of insulin resistance and type 2 diabetes on lipoprotein subclass particle size and concentration determined by nuclear magnetic resonance. Diabetes 2003;52(2):453-62.

20. Howard BV, Pan XR, Harper I, Kuusi T, Taskinen MR. Lack of sex differences in high density lipoproteins in Pima Indians. Studies of obesity, lipase activities, and steroid hormones. Arteriosclerosis 1987;7(3):292-300.

21. Pitsavos C, Panagiotakos DB, Skoumas J, Papadimitriou L, Stefanadis C. Risk stratification of the apolipoprotein $\mathrm{B}$, apolipoprotein A1, and apolipoprotein B/A1 ratio on the prevalence of the metabolic syndrome: the ATTICA study. Angiology 2008;59(3):335-41. 